\title{
nature
}

\section{Obstacles to biodefence}

The United States is expected soon to establish its Department of Homeland Security. The precise threats facing the nation are uncertain, but challenges in its preparedness to deal with attacks on health and agriculture are all too obvious.

n late 1999, US, European and Japanese semiconductor and information-technology businesses were severely damaged, losing revenues and profits and raising prices to compensate for a sudden shortfall in the availability of semiconductors and other components. The cause was an earthquake in Taiwan, which not only led to a tragic loss of life, but also disrupted suppliers of $10 \%$ of the world's silicon processing capacity and more than half of the world's semiconductor manufacturing, and suppliers of $80 \%$ of the motherboards used in computer manufacturing. Miraculously, Taiwan recovered its capacity to export within two weeks. This episode, however, illustrates the exposure of countries to remote sources of disruption, amplifying the obvious point that the ways by which any country could be threatened by terrorists are innumerable.

One year on from the attacks on the World Trade Center and the Pentagon, the United States is on the threshold of establishing a new federal-government Department of Homeland Security (see page 10). As discussed at a meeting organized by the Johns Hopkins Center for Civilian Biodefense Strategies in Baltimore, Maryland, last month, the lack of clarity of possible threats is only one of the problems facing the new department. The meeting's participants, who hold senior positions in the key agencies and the research community, emphasized that the challenge to the department from unpredictability is compounded by a woeful lack of national preparedness for biological attacks, both natural and artificial. Can the world responsibly go on assuming that non-biological terrorist threats are more probable?

The potential dual use - therapeutic and destructive - of developments in biological understanding is all too apparent. It highlights the need to focus all the more on basic biomedical research. Knowledge of the genomes of strains of anthrax and structures of its receptors poses little threat in practice, but understanding the immune system is another story. We are not far away from determining critical nodes in the molecular networks of immunity and its control, and of other key biological circuits. Understanding these will be crucial for overcoming diseases but might greatly simplify the tasks of terrorists. Such networks could be attacked not only by modified pathogens but also by small molecules: biological and chemical weapons should be treated as distinct but parallel paths in the development of defences.

\section{Lack of understanding}

The Bush administration has recommended that billions of dollars be distributed across the federal budget for 2003 to combat terrorist threats. Turf wars and battles between politicians over the regional allocation of funds will abound, and champions of various technologies will compete with each other within and beyond the Department of Defense. According to participants at Baltimore - many of whom have considerable experience of working with military staff — one of the greatest problems is a mind-set in defence circles that is steeped in a history of dealing with nuclear threats. There is a persistent lack of appreciation of just how different biological attacks are to traditional threats, compounded by a lack of biological literacy and awareness.

The capacity of countries to respond even to natural infections is weak. In particular, the US Department of Health and Human
Services' ability to acquire vaccines was described by one informed participant as a failure. Moreover, vaccine suppliers come in many nationalities, so here, as in many other aspects of bioterrorist threats, international coordination poses a further set of challenges for which no adequate framework is in place. Again, the leadership in vaccine provision suffers from a lack of scientific understanding. There has not been enough speaking out by learned societies on this issue.

The lack of relevant pharmaceutical capacity could be seen as a sign that the market is incapable of catering for vaccine provision for healthcare, let alone for biodefence. Yet in the longer term, the threat of disruption to business - as illustrated by the Taiwanese earthquake - suggests that there is an underlying market incentive. Could that be turned into investment in a new biodefence industry? One can only be sceptical in the face of the enormous range of possible threats. And the incentives are even weaker in the agricultural biotech industry, where profit margins are smaller than in health, and in which government appears to be even less prepared.

\section{Rapid detection}

A lack of preparedness is also mirrored in a lack of sophistication in specifying and maintaining the defensive technology to operational standards, specifically in diagnostics and sensors (see page 8). As described at the meeting, for decades, and especially over the years since the Gulf War ended in 1991, the US government appears to have been deluded in its optimistic faith in the ability of current sensor technologies to help protect the population. Specifications that need to be met are weak, and claims by suppliers are rarely adequately scrutinized.

One priority over the next five years must be to develop a capacity to deliver prompt detection and diagnosis following a chemical or biological attack, along with an ability to mobilize protection and treatment quickly. The latter requires a mix of private and public industrial investment incentives to be developed with high priority, so that pharmaceutical companies would each have two or three related programmes under way, and tens of biotechnology companies would also be developing products. Easily said, but the additional investment required would amount to much more than a billion dollars over several years.

One way of improving scientific and technical awareness of the problems inherent in biological defence, and developing appropriate capacity to respond, is to have key stakeholders collaborate in the development of scenarios of imaginary attacks, however speculative. This process will have the additional benefit of putting under scrutiny the performance of technologies and improving the standards to be applied in manufacture, as well as the ability of leaders to summon up the assistance and information they require in prioritizing their response. These studies would need to include attacks using smallpox or anthrax aerosols, and botulinum toxin in food. Scenarios of this kind have been studied on occasion, and the process can drown in a plethora of competing assumptions and models, so it needs good management. But the lack of national preparedness and high-level appreciation of biological threats suggests that there is more to be done in using such an approach. 